\title{
DEPÓSITO DE PULVERIZAÇÃO COM DIFERENTES PADRÕES DE GOTAS EM APLICAÇÕES NA CULTURA DO ALGODOEIRO
}

\section{REGINALDO T. DE SOUZA ${ }^{1}$, ROGÉRIO D. DE CASTRO ${ }^{2}$, LUIZ A. PALLADINI ${ }^{3}$}

\begin{abstract}
RESUMO: Com os objetivos de avaliar a deposição da calda de pulverização sobre plantas de algodão e a eficiência da pulverização com diferentes padrões de gotas sob condições adversas de temperatura e umidade, realizou-se um experimento para a determinação dos volumes de depósitos da pulverização no final de ciclo da cultura. $\mathrm{O}$ trabalho foi executado com pulverizador automotriz, equipado com pontas JA3 (jato cônico vazio) e jato plano com indução a ar AD-IA/D 11003 (duplo), AD-IA 11003 que, na pressão trabalhada, proporcionaram volume de aplicação de $150 \mathrm{~L}^{-1} \mathrm{~h}^{-1}$, e a ponta AD-IA 11004 que, na mesma pressão, proporcionou volume aplicado de $200 \mathrm{~L} \mathrm{ha}^{-1}$. Para a determinação dos volumes depositados nas folhas do terço superior, médio e inferior das plantas de algodão, utilizou-se do corante alimentício azul brilhante adicionado à calda de pulverização, na concentração de $0,179 \%$. Após a pulverização, as folhas foram lavadas, e o volume recuperado foi analisado por espectrofotometria. Os resultados obtidos foram submetidos à análise de regressão pelo modelo de Gompertz para a comparação entre os tratamentos. A ponta AD-IA 11003 proporcionou os maiores volumes de depósito nas diferentes posições da planta, e a eficiência da aplicação foi de 27,45; 35,08; 47,60 e 39,25\% para as pontas JA3, AD-IA/D 11003, AD-IA 11003 e AD-IA 11004, respectivamente.
\end{abstract}

PALAVRAS-CHAVE: deposição, traçantes, algodão.

\section{SPRAY LEAF DEPOSITION FROM DIFFERENT NOZZLES AND DROP PATTERNS IN LATE SEASON APPLICATIONS ON COTTON PLANTS}

\begin{abstract}
The experiment was carried out to evaluate the leaf deposition and the efficiency of different drop pattern by spraying cotton plants at the end of the growing season, under adverse air temperature and humidity conditions. The sprays, applied at the same pressure, were made with a JACTO UNIPORT speed-sprayer equipped with the nozzles JA3 (hollow cone), AD-IA/D 11003 (double flat fan), and AD-IA 11003 (flat fan) for $150 \mathrm{~L} \mathrm{ha}^{-1}$ spray volume, and the nozzle AD-IA 11004 (flat fan) for $200 \mathrm{~L} \mathrm{ha}^{-1}$ spray volume. It was applied the FD\&C Brue $\mathrm{n} .1$ tracer at $0.179 \%$ in the spraying solution to determine the volume of spray deposition on leaf samples obtained from the upper, the middle, and the lower third of the plants canopy. The leaf samples were washed with $20 \mathrm{ml}$ of deionized water. The recovered volumes were analyzed by spectrophotometry. The data were submitted to the Gompertz model regression analysis for treatments comparison. The nozzle AD-IA 11003 gave the highest leaf deposit in all plant parts. The application efficiency was 27.45; 35.08; 47.60 and $39.25 \%$ for the nozzles JA3, AD-IA/D 11003, AD-IA 11003, and AD-IA 11004, respectively.
\end{abstract}

KEYWORDS: tracers, leaf deposition, cotton.

\footnotetext{
${ }^{1}$ Eng ${ }^{\mathrm{o}}$ Agrônomo, Pesquisador Doutor, Embrapa Uva e Vinho, Estação Experimental de Viticultura Tropical, Jales - SP, recco@cnpuv.embrapa.br

${ }^{2}$ Engo Agrônomo, Professor Mestre, UNIVAG, Instituto E. Matogrossense, Várzea Grande - MT.

${ }^{3}$ Eng ${ }^{0}$ Agrônomo, Pesquisador Doutor, EPAGRI, Caçador - SC.

Recebido pelo Conselho Editorial em: 21-9-2005

Aprovado pelo Conselho Editorial em: 19-9-2006
} 


\section{INTRODUÇÃO}

A cultura do algodão é atualmente uma das mais importantes do mundo pela ampla utilização nos diversos segmentos e setores agroalimentícios e industriais. Entre os países produtores, o Brasil vem se destacando pelo aumento de sua produção devido, entre outros fatores, à abertura de mercado que possibilitou a introdução de novas máquinas de semeadura e colheita, ampliando, dessa forma, a área cultivada, principalmente no Centro-Oeste brasileiro.

Condições de estresse térmico, hídrico ou de radiação solar, que ocorram principalmente na fase reprodutiva da cultura, podem acarretar situações de desequilíbrio fisiológico na planta (OOSTERHUIS, 1999; BELTRÃO \& SOUZA, 2001), tornando-a mais vulnerável ao ataque de pragas e doenças, que influenciam na qualidade dos frutos e dos capulhos (SOARES \& SILVA, 1999). Sob tais condições meteorológicas, aplicações necessárias para o controle podem ser ineficientes pelo uso inadequado de equipamentos de pulverização, principalmente no que se refere ao volume de aplicação e padrão de gota.

A qualidade da cobertura do alvo está condicionada ao diâmetro de gotas. As de menor diâmetro proporcionam maior penetração entre as folhas das culturas (CHRISTOFOLETTI, 1992; SANTOS, 1992; OZEKI \& KUNZ, 1998), entretanto, ao se reduzir o diâmetro das gotas para aumentar a cobertura do alvo, com o mesmo volume de aplicação, maior é a interferência do vento, causando deriva, e da temperatura e umidade do ar, causando perdas por evaporação (CHRISTOFOLETTI, 1999).

CUNHA et al. (2003), ao estudarem o potencial de deriva das pontas de jato plano comum API 11002, com e sem óleo vegetal, e ADI 11002 (antideriva), verificaram que as pontas antideriva e a adição de adjuvante permitiram controle da deriva, reduzindo o arrastamento de partículas e diminuindo significativamente o número de gotas fora da área-alvo. SUMNER \& SUMNER (1999) constataram a redução do potencial de deriva com a utilização de pontas antideriva.

Para MATUO (1998), o produto fitossanitário deve exercer sua ação sobre determinado organismo, portanto é necessário que o alvo seja atingido; sendo assim, qualquer quantidade de produto químico (ou agente de outra natureza) que não atinja o alvo, não terá qualquer eficácia e estará representado como forma de perda. Portanto, é preciso conhecer o espectro das gotas pulverizadas, de forma a adequar o seu tamanho, garantindo, ao mesmo tempo, eficácia biológica e segurança ambiental, de acordo com as condições meteorológicas no momento da aplicação (CUNHA et al., 2003).

Dessa forma, os objetivos deste trabalho foram avaliar a deposição obtida na pulverização em plantas de algodão e a eficiência da pulverização com diferentes padrões de gotas, sob condições adversas de temperatura e umidade.

\section{MATERIAL E MÉTODOS}

Para a realização da pulverização, foi utilizado um pulverizador automotriz, na velocidade de $10 \mathrm{~km} \mathrm{~h}^{-1}$, com as seguintes características: tanque de 2.000 litros, de fibra de vidro, bomba com capacidade de vazão para $150 \mathrm{~L} \mathrm{~min}^{-1}$, barras de $21 \mathrm{~m}$ com acionamento hidráulico, equipados com 43 porta-bicos Bijet com válvula antigotejo e os demais tipos de pontas e pressão de trabalho, marca, tipo de jato e volume de calda, conforme descritos na Tabela 1.

A solução traçadora adicionada à calda foi constituída do corante Azul Brilhante (FD\&C Blue n.1) catalogado internacionalmente pela Food, Drug \& Cosmetic, a 0,179\%, e o pigmento fluorescente Saturn Yellow, também a $0,179 \%$, solubilizado com Vixilperse a $0,015 \%$, conforme metodologia descrita por PALLADINI (2000). 
TABELA 1. Tipos de pontas, marca, tipo de jato, pressão de trabalho e volume de aplicação e espectro de gotas, utilizados nos tratamentos.

\begin{tabular}{|c|c|c|c|c|c|c|}
\hline Tratamento & $\begin{array}{l}\text { Tipo de } \\
\text { Ponta }\end{array}$ & Marca & $\begin{array}{l}\text { Tipo de } \\
\text { Jato }\end{array}$ & $\begin{array}{c}\text { Pressão } \\
(\mathrm{kPa})\end{array}$ & $\begin{array}{c}\text { Volume de } \\
\text { Aplicação }\left(\mathrm{L} \mathrm{ha}^{-1}\right)\end{array}$ & Espectro de Gotas \\
\hline 1 & JA-3 & Jacto & Cone vazio & 732 & 150 & Finas \\
\hline $2 * *$ & AD-IA/D 11003 & Mag & Jato & 317 & 150 & Grossas \\
\hline $3 * *$ & AD-IA 11003 & Mag & Jato plano & 317 & 150 & Muito grossas \\
\hline $4 * *$ & AD-IA 11004 & Magno & Jato plano & 317 & 200 & Muito /extr. gr.* \\
\hline
\end{tabular}

*Muito grossas/extremamente grossas; **pontas com indução de ar.

No momento da aplicação, a temperatura do ar estava em $31{ }^{\circ} \mathrm{C}$, umidade relativa do ar de $33 \%$ e velocidade do vento a $7 \mathrm{~km} \mathrm{~h}^{-1}$. Os diferentes tratamentos foram aplicados em aproximadamente 30 minutos em área equivalente a $10,5 \mathrm{~m}$ de largura e $50 \mathrm{~m}$ de comprimento. Os alvos naturais foram constituídos por folhas de algodão coletadas aleatoriamente em três níveis das plantas (terço superior, médio e inferior), com 100 repetições por tratamento.

Após a aplicação, as folhas foram coletadas e armazenadas individualmente, em sacos plásticos. Em seguida, as amostras foram transportadas para o laboratório, para a remoção do depósito com $20 \mathrm{~mL}$ de água deionizada. A solução da lavagem foi armazenada em recipientes de vidro âmbar, para posterior determinação quantitativa do depósito do traçante. As amostras foram selecionadas no laboratório para que os diferentes tratamentos apresentassem o mesmo intervalo de área foliar, segundo metodologia preconizada por SOUZA (2002).

A determinação da quantidade do traçador depositada, em cada amostra, foi realizada com espectrofotômetro no comprimento de onda de $630 \mathrm{~nm}$ para o azul brilhante. Os dados foram transformados em mg L $\mathrm{m}^{-1}$, de acordo com coeficiente angular da curva-padrão da calda contendo o traçante. As concentrações em $\mathrm{mg} \mathrm{L}^{-1}$ foram transformadas em volume por unidade de área $\left(\mu \mathrm{L} \mathrm{cm}^{-2}\right)$.

Os valores dos depósitos, em percentagem, foram ajustados pelo modelo de Gompertz.

\section{RESULTADOS E DISCUSSÃO}

Na Figura 1, estão apresentadas as freqüências acumuladas representadas pela percentagem de folhas amostradas em função dos volumes de depósito por unidade de área foliar. Observa-se que os volumes de depósitos nas folhas pulverizadas com volume de calda de $150 \mathrm{~L} \mathrm{ha}^{-1}$ com as pontas JA 3 (cone vazio), AD-IA/D 11003 (jato plano duplo) e AD-IA 11003 (jato plano) foram obtidos com padrão de gotas finas, grossas e muito grossas, respectivamente. Sendo assim, o aumento no tamanho de gotas reduziu as perdas por evaporação e deriva (exoderiva), e, conseqüentemente, maior volume ficou retido nas folhas do terço superior da planta. Em geral, gotas finas estão mais sujeitas à perda por deriva e evaporação, diminuindo a eficiência da pulverização e aumentando os riscos de contaminação ambiental (SALYANI, 1999; SUMMER \& SUMMER,1999), principalmente sob condições adversas de temperatura e umidade. KIRK et al. (1992) verificaram altos níveis de depósitos de pulverização na cultura do algodoeiro em aplicações sob condições de baixa temperatura, baixa velocidade do vento e alta umidade relativa do ar.

A ponta AD-IA 11004, que na mesma pressão das demais pontas de jato plano aplicou volume de calda de $200 \mathrm{~L} \mathrm{ha}^{-1}$, produzindo gotas classificadas como muito grossas/extremamente grossas, não proporcionou aumento de depósito nas folhas na mesma proporção do aumento de volume de aplicação em relação à ponta do mesmo tipo (AD-IA 11003), provavelmente pela não-retenção da calda pulverizada na parte superior da planta. O escorrimento (endoderiva) ou a pulverização não proporcionou número de gotas suficiente para aumentar a cobertura do alvo, mesmo com o aumento do volume aplicado. DERKSEN \& SANDERSON (1996) avaliaram a influência do volume de calda na deposição foliar de agrotóxicos e verificaram, entretanto, que o uso de altos volumes de aplicação proporcionou melhor cobertura e menores variações de deposição ao longo do dossel. 


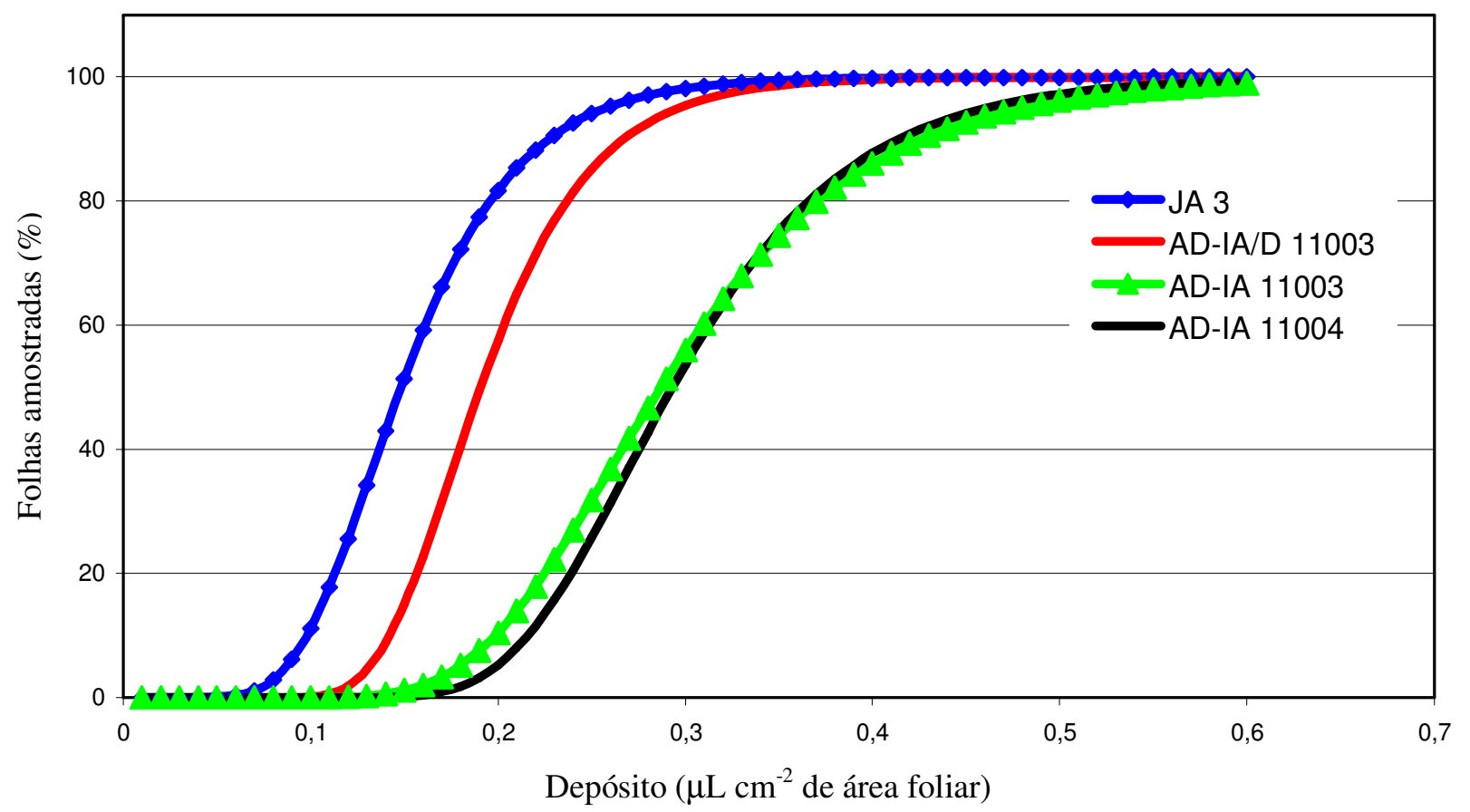

FIGURA 1. Frequiências acumuladas representadas pela percentagem de folhas amostradas no terço superior da planta do algodoeiro, em função dos volumes de depósitos por unidade de área foliar produzidos por diferentes pontas de pulverização.

Ao analisar o depósito da pulverização nas folhas do terço médio da planta, apresentados na Figura 2, verificam-se as mesmas tendências apresentadas para o terço superior da planta; volumes maiores depositados para maior tamanho das gotas produzidas, com exceção das pontas de jato plano AD-IA 11003 e AD-IA 11004, nas quais o maior volume aplicado proporcionou maior volume depositado em aproximadamente $80 \%$ das folhas. Por outro lado, com volume depositado próximo a $20 \%$ nas folhas que receberam menor depósito e, se esse intervalo naturalmente representar a maior possibilidade de escape ao controle de determinado inseto ou fungo, os níveis de controle seriam os menores com a ponta AD-IA 11004, pois os volumes depositados não foram proporcionais ao aumento no volume aplicado. Portanto, o que realmente determinaria o controle, seria a concentração de ativo na calda que estaria mais diluída na aplicação a $200 \mathrm{~L} \mathrm{ha}^{-1}$. Dessa forma, a possibilidade de escape ao controle seria pelo menos 33\% maior em relação ao AD-IA 11003. Sendo assim, os volumes de depósitos, mesmo em condições ambientais adversas para a aplicação dos produtos, aumenta até certo ponto com o aumento no tamanho das gotas e, acima disso, mesmo com o aumento de volume, a deposição é prejudicada. Isso foi observado por REED \& SMITH (2001), ao avaliarem a mortalidade de Helilothis virensens por inseticidas aplicados com diferentes volumes de aplicação e padrões de gotas. Os autores verificaram maior penetração no dossel das plantas com gotas pequenas, e a mortalidade de lagartas tendeu a diminuir com o aumento de volume aplicado, sugerindo que a concentração de ativo seria um fator que interfere na mortalidade, pois esse fator é inversamente proporcional ao volume de aplicação.

As folhas localizadas no terço inferior das plantas representam o alvo mais difícil de atingir em pulverizações. A sobreposição das folhas existentes no caminho da gota e, também, a maior possibilidade de perda por evaporação ou deriva das gotas menores, no espectro formado de acordo com as características de cada ponta, em condições ambientais adversas e pela maior distância percorrida, implicam menor número de gotas chegando ao alvo e irregularidade no volume depositado. 
Isso pode ser observado na Figura 3, em que os volumes depositados em função das frequiências acumuladas, representadas pela percentagem de folhas amostradas, não apresentaram o mesmo comportamento em relação às demais partes da planta. Verifica-se que a ponta AD-IA 11003 proporcionou maior volume depositado até $50 \%$ das folhas que menos receberam depósito em relação à ponta ADIA/D 11003 e, ao se analisar a população amostrada, observa-se que a primeira proporcionou espectro mais homogêneo, ou seja, menores foram as diferenças entre os maiores e menores depósitos nas folhas.

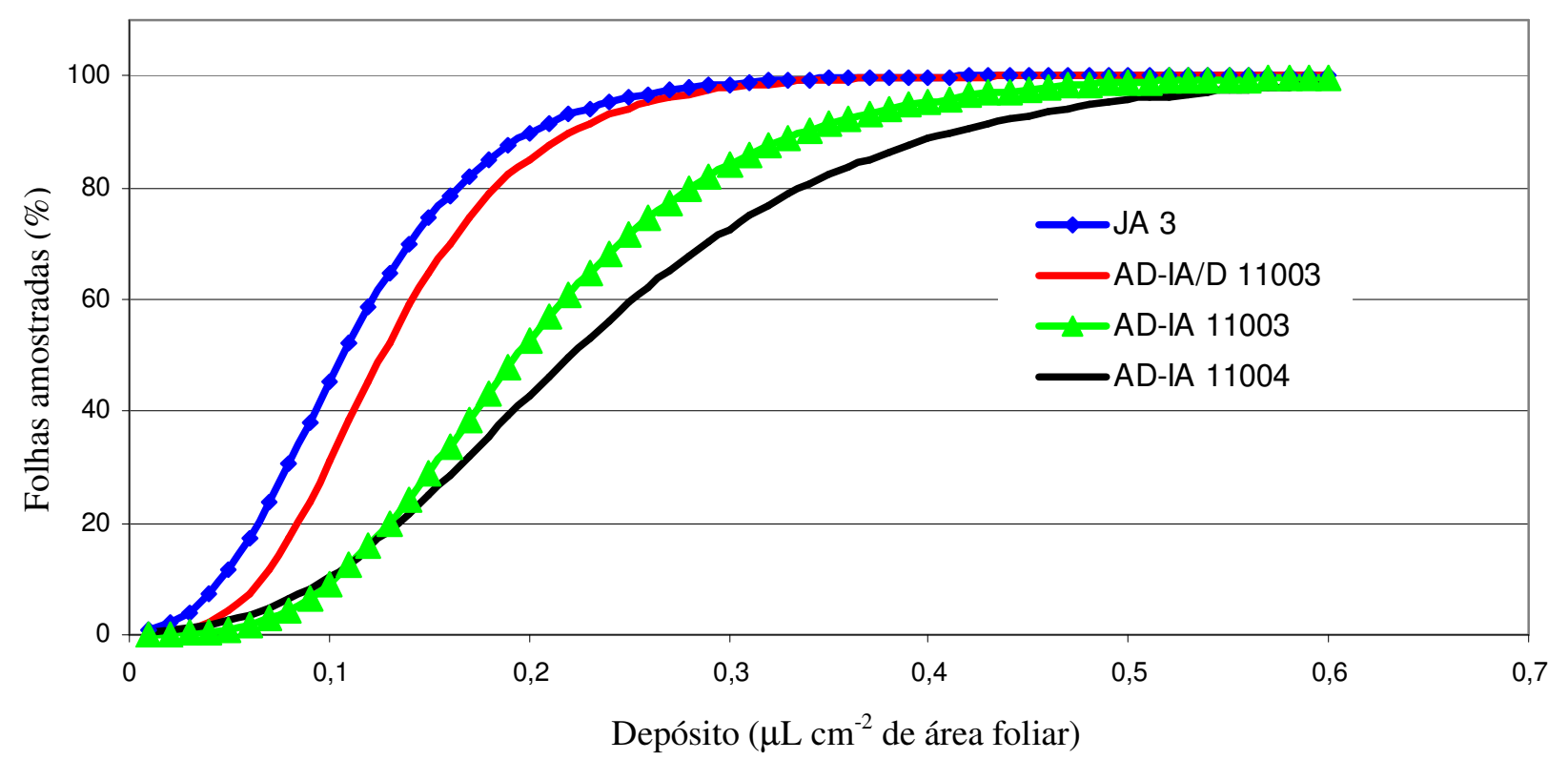

FIGURA 2. Freqüências acumuladas representadas pela percentagem de folhas amostradas no terço médio da planta do algodoeiro, em função dos volumes de depósitos por unidade de área foliar obtidos por diferentes pontas de pulverização.

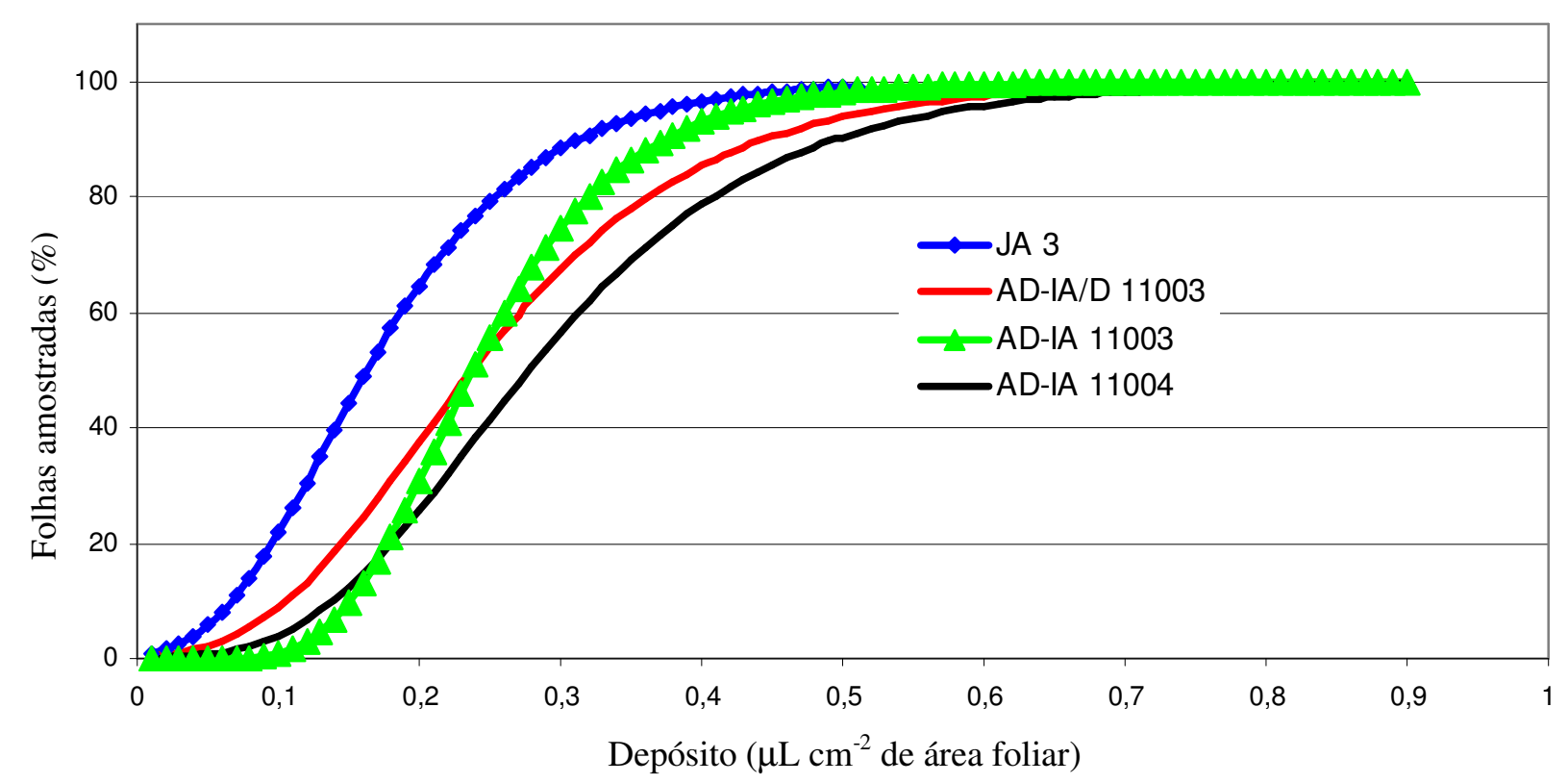

FIGURA 3. Frequiências acumuladas representadas pela percentagem de folhas amostradas no terço inferior da planta de algodão, em função dos volumes de depósitos por unidade de área foliar produzidos por diferentes pontas de pulverização. 
Conhecendo-se a área foliar média das plantas nas diferentes posições, foi possível calcular o volume retido por hectare proporcional ao volume aplicado para as diferentes pontas e, dessa forma, expressar numericamente, pelos resultados apresentados na Tabela 2, a eficiência da aplicação. Pode se verificar aumento nas quantidades retidas pela planta com o aumento do padrão de gotas produzidas pelas diferentes pontas no volume de calda de $150 \mathrm{~L} \mathrm{ha}^{-1}$, chegando a $47,6 \%$ para a ponta AD-IA, aproximadamente $1,7 \mathrm{vez}$ superior à ponta JA 3. Observa-se, também, que a eficiência diminuiu com o aumento no tamanho de gotas e volume ao se compararem os resultados com pontas do mesmo tipo e vazões diferentes (AD-IA 11003 e 11004). Portanto, existe um limite no aumento do tamanho das gotas para ser eficientemente captadas pelo alvo, mesmo em condições adversas de umidade e temperatura no momento da aplicação.

TABELA 2. Eficiência de aplicação e volume de depósito de pulverização nas diferentes partes da planta proporcionados pelas pontas JA 3, AD-IA/D 11003 e AD-IA 11003 com volume de calda a $150 \mathrm{~L} \mathrm{ha}^{-1}$ e AD-IA 11004 com volume de calda a $200 \mathrm{~L} \mathrm{ha}^{-1}$.

\begin{tabular}{lccccc}
\hline \multicolumn{1}{c}{ Posição } & Área Foliar & \multicolumn{3}{c}{ Depósito $\left(\mathrm{L} \mathrm{ha}^{-1}\right)$} \\
\cline { 3 - 6 } na Planta & $\left(\mathrm{cm}^{2}\right)$ & JA 3 & AD-IA/D 11003 & AD-IA 11003 AD-IA 11004 \\
\hline Terço inferior & 581,77 & 10,54 & 14,95 & 14,63 & 17,76 \\
Terço médio & $1.314,40$ & 15,17 & 18,13 & 27,33 & 30,77 \\
Terço superior & $1.015,31$ & 15,47 & 19,54 & 29,44 & 29,97 \\
\hline Total & $2.911,48$ & 41,17 & 52,62 & 71,41 & 78,50 \\
\hline Eficiência da aplicação(\%) & & 27,45 & 35,08 & 47,60 & 39,25 \\
\hline
\end{tabular}

No terço inferior da planta, verifica-se que o valor médio de depósitos entre as pontas ADIA/D 11003 e AD-IA 11003 apresentaram valores semelhantes (Tabela 2). No entanto, ao comparar a dispersão do volume depositado na população de folhas apresentados na Figura 3, verificam-se diferenças no volume acumulado até $50 \%$ das folhas que receberam maior ou menor depósito, demonstrando que a metodologia empregada para a avaliação das diferentes tecnologias empregadas na pulverização, detecta variações que a análise por testes de médias não permitiria. PERGHER et al. (1997), ao testarem diferentes pulverizadores, afirmaram que essa metodologia permite concentrar a análise na porção de área foliar com os mais baixos depósitos. COATES (1996), ao testar o controle de mosca-branca e deposição de inseticidas em aplicações com pulverizadores eletrostáticos e gotas controladas, comparados com depósitos de bicos hidráulicos, concluiu que a variação dos depósitos foi elevada, para verificação das diferenças na deposição ou mortalidade de insetos entre tipos de bicos, e esses resultados contraditórios indicariam que o desenvolvimento de uma recomendação por tamanho ideal de gotas e volume de aplicação seria importante.

\section{CONCLUSÕES}

O aumento no tamanho da gota, até certo limite, melhora a eficiência da pulverização sob condições adversas de umidade e temperatura, entretanto a deposição no alvo em pulverizações com pontas que produzem gotas extremamente grossas é prejudicada, mesmo com o aumento do volume de aplicação.

Pontas de jato plano com indução de ar, caracterizadas pela produção de gotas grossas, proporcionaram os maiores volumes de depósitos nas folhas e maior uniformidade da pulverização nos diferentes níveis da planta.

A variabilidade dos depósitos nas folhas dos níveis superior, mediano e inferior da planta aumenta do ápice para a base. 


\section{AGRADECIMENTOS}

FACUAL - Fundo de Apoio a Cultura do Algodão e COOPERFIBRA.

\section{REFERÊNCIAS}

BELTRÃO, N.E. de M.; SOUZA, J.G. de. Fisiologia e ecofisiologia do algodoeiro. In: EMBRAPA AGROPECUÁRIA OESTE. Algodão: tecnologia de produção. Dourados: Embrapa Agropecuária Oeste, 2001. 296 p.

CHRISTOFOLETTI, J. C. Considerações sobre deriva nas pulverizações agrícolas e seu controle. São Paulo: TeeJet Soth América, 1999. 15 p. (Boletim Técnico BT-04/99).

CHRISTOFOLETTI, J. C. Manual Shell de máquinas e técnicas de aplicação de defensivos. São Paulo: Shell Brasil, 1992. 122 p.

COATES, W.E. Spraying technologies for cotton: deposition and efficacy. Applied Engeneering in Agriculture, St. Joseph, v.12, n.3, p.287-96, 1996.

CUNHA, J.P.A.R.; TEIXEIRA, M.M.; COURY, J.R.; FERREIRA, L.R. Avaliação de estratégias para redução da deriva de agrotóxicos em pulverizações hidráulicas. Planta Daninha, Viçosa-MG, v.21, n.2, p.325-32, 2003.

DERKSEN, R.C.; SANDERSON, J.P. Volume, speed and distribution technique effects on poinsentia foliar deposits. Transactions of the ASAE, St. Joseph, v.39, n.1, p. 5-9, 1996.

KIRK, I.W.; BOUSE, L.F.; CARLTON, J.B.; FRANZ, E.; STERMER, R.A. Aerial spray deposition in cotton. Transactions of the ASAE, St. Joseph, v.32, n.5, p.1393-9, 1992.

MATUO, T. Fundamentos da tecnologia de aplicação de agrotóxicos. In: TECNOLOGIA E SEGURANÇA NA APLICAÇÃO DE AGROTÓXICOS: NOVAS TECNOLOGIAS, 2., 1998, Santa Maria. Anais... Santa Maria: Departamento de Defesa Fitossanitária; Sociedade de Agronomia de Santa Maria, 1998. p.95-101.

OOSTERHUIS, D. M. Growth and development of a cotton plant. In: CIA, E.; FREIRE, E.C.; SANTOS,W.J. dos. Cultura do algodoeiro. Piracicaba: POTAFOS, 1999. 286 p.

OZEKI, Y.; KUNZ, R.P. Tecnologia de aplicação aérea - aspectos práticos. In: Tecnologia e segurança na aplicação de agrotóxicos: novas tecnologias. Santa Maria: Departamento de Defesa Fitossanitária/UFMS, 1998. p.65-78.

PALLADINI, L.A. Metodologia para avaliação da deposição em pulverizações. 2000. $111 \mathrm{f}$. Tese (Doutorado em Proteção de Plantas) - Faculdade de Ciências Agronômicas, Universidade Estadual Paulista, Botucatu, 2000.

PERGHER, G.; GUBIANI, R.; TONETTO, G. Foliar deposition and pesticide losses from three airassisted sprayers in a hedgerow vineyard. Crop Protection, Oxford, v.16, n.1, p.24-33, 1997.

REED. J.T.; SMITH, D.B. Droplet size and spray volume effects on insecticide deposit and mortality of heliothine (Lepidoptera: noctuidae) larvae in cotton. Journal of Economic Entomology, Lanham, v.94, n.3, p.640-7, 2001.

SALYANI, M. Optimization of sprayer output at different volume rates. St. Joseph: ASAE, 1999. 16 p. (ASAE, Paper No. 99-1028).

SANTOS, J.M.F. Aviação agrícola - Manual de tecnologia de aplicação de agroquímicos. São Paulo: Rhodia Agro, 1992. 100 p. 
SOARES, J.J.; SILVA, E.Q. da. Efeito do ataque de Alabama argillacea no desenvolvimento vegetativo e sua relação com a fenologia do algodoeiro. Campina Grande: Embrapa-CNPA, 1999. 7 p. (Comunicado Técnico, 100).

SOUZA, R.T. Efeito da eletrização de gotas sobre a variabilidade dos depósitos de pulverização e eficácia do glyphosate no controle de plantas daninhas da cultura da soja. 2002 . $69 \mathrm{f}$. Tese (Doutorado em Agricultura) - Faculdade de Ciências Agronômicas, Universidade Estadual Paulista, Botucatu, 2002.

SUMNER, P.E.; SUMNER, S.A. Comparison of new drift reduction nozzles. St. Joseph: ASAE, 1999. 17 p. (ASAE Paper n.99-1156). 